PROCEEDINGS OF THE

AMERICAN MATHEMATICAL SOCIETY

Volume 131, Number 6, Pages 1943-1951

S 0002-9939(02)06809-0

Article electronically published on November 14, 2002

\title{
A COCYCLE FORMULA FOR THE QUATERNIONIC DISCRETE SERIES
}

\author{
ROBERT W. DONLEY, JR.
}

(Communicated by Rebecca Herb)

\begin{abstract}
Schmid's proof of the Kostant-Langlands conjecture for discrete series representations of a semisimple Lie group provides a Hilbert space realization of such representations in $L^{2}$-cohomology. We give an explicit description of these harmonic forms for the quaternionic discrete series.
\end{abstract}

\section{INTRODUCTION}

Let $G$ be a connected semisimple Lie group with finite center, let $K$ be a maximally compact subgroup, and suppose $\operatorname{rank} G=\operatorname{rank} K$. Then $G$ possesses discrete series representations $[\mathrm{HC}$; that is, $G$ has irreducible unitary representations with square-integrable matrix coefficients.

A realization for such representations was conjectured independently by Kostant and Langlands using methods of complex analysis. This conjecture was proved by Schmid in a series of papers ending with $\mathrm{Sc}$.

Let $\mathfrak{g}_{0}$ be the Lie algebra of $G$, and let $\mathfrak{g}$ be its complexification. Real Lie algebras are denoted with a zero subscript; the complexification omits the subscript. Choose a Cartan subgroup $T$ in $K$. Let $\mathfrak{t}_{0}$ be the Lie algebra of $T$, and form the set of roots $\Delta=\Delta(\mathfrak{g}, \mathfrak{t})$. If one chooses a positive root system $\Delta^{+}$in $\Delta$, there is an associated triangular decomposition $\mathfrak{g}=\mathfrak{t}+\mathfrak{n}+\mathfrak{n}^{\prime}$, where $\mathfrak{n}$ (resp. $\left.\mathfrak{n}^{\prime}\right)$ is the direct sum of root spaces associated to negative (resp. positive) roots. Let $\mathfrak{b}=\mathfrak{t}+\mathfrak{n}$.

With this choice, the homogeneous space $G / T$ inherits a $G$-invariant complex structure; the antiholomorphic tangent space at $e T$ is identified with $\mathfrak{n}$. One may construct a holomorphic homogeneous holomorphic vector bundle $\mathcal{L}_{\lambda}$ on $G / T$ and its associated geometric constructions (i.e. Dolbeault cohomology); see section 1 for details. To construct representations on these spaces, $G$ acts by left-translation $L$.

Let $\bar{\partial}$ be the Cauchy-Riemann operator, and let $\bar{\partial}^{*}$ be its formal adjoint with respect to a certain invariant inner product on $\mathfrak{n}$. A form $\omega$ is called strongly harmonic if it lies in both Ker $\bar{\partial}$ and Ker $\bar{\partial}^{*} ; \omega$ is called harmonic if it lies in the kernel of the associated Laplacian $\square=\bar{\partial} \bar{\partial}^{*}+\bar{\partial}^{*} \bar{\partial}$. Explicit formulas for $\bar{\partial}$, $\bar{\partial}^{*}$ and $\square$ in terms of a root basis occur in [GS].

Received by the editors January 22, 2002.

2000 Mathematics Subject Classification. Primary 22E46, 53C65.

Key words and phrases. Quaternionic discrete series, harmonic forms, matrix coefficients.

The author was supported by MSRI. Research at MSRI was supported in part by NSF grant DMS-9810361. 
Theorem $0.1(\underline{\mathrm{Sc}})$. Suppose $\lambda+\delta$ is analytically integral, $s=\operatorname{dim}_{\mathbb{C}} K / T$, and $\mathcal{H}_{2}^{0, s}\left(G / T, \mathcal{L}_{\lambda}\right)$ is the space of square-integrable, strongly harmonic $(0, s)$-forms on $G / T$ with values in $\mathcal{L}_{\lambda}$. If $\lambda+\delta$ lies in the negative Weyl chamber, then $\left(L, \mathcal{H}_{2}^{0, s}\left(G / T, \mathcal{L}_{\lambda}\right)\right)$ gives a unitary realization of the discrete series representation $(\pi, V)$ of $G$ with underlying Harish-Chandra module $A^{\mathfrak{b}}(\lambda)$.

In this case, the conditions of harmonic and strongly harmonic are equivalent.

One wishes to give an explicit description of forms arising in this manner. [Ba] constructs strongly harmonic cocycles associated to an invariant indefinite form; the resulting space is sufficient for describing the associated Dolbeault cohomology classes, but square integrability of such forms remains an open question.

We recall the methods of [Do]. An explicit harmonic representative for the associated (one-dimensional) $\mathfrak{n}$-cohomology group for the $K$-finite vectors of $V^{*}$ yields an intertwining operator from any other realization of $V$ into $\mathcal{H}_{2}^{0, s}\left(G / T, \mathcal{L}_{\lambda}\right)$. Geometric considerations imply that this $\mathfrak{n}$-cohomology class may be described entirely in terms dependent on a fixed nonzero lowest weight vector of the minimal $K$-type of $V$.

In this work, an explicit description of this class is given for the quaternionic discrete series of $\mathrm{GW}$ and Go. Since the resulting forms are described using matrix coefficients, they are automatically square-integrable. The main results are Theorem 3.2 and Corollary 3.3.

We remark that our methods provide an algebraic proof for existence of cohomology; see [Do] for the case of discrete series limits.

\section{Preliminaries}

Let $\mathfrak{g}_{0}=\mathfrak{k}_{0}+\mathfrak{p}_{0}$ be the Cartan decomposition determined by $K$. A root $\alpha$ is called compact (resp. noncompact) if the root space $\mathfrak{g}_{\alpha}$ is contained in $\mathfrak{k}$ (resp. $\mathfrak{p})$. Let $B$ be the Killing form of $\mathfrak{g}$; we also denote it and the induced form on $\mathfrak{g}^{*}$ by parentheses. Results will be phrased in notation determined by the following proposition.

Proposition 1.1 (cf. [He], Ch. III; $[\mathrm{Kn}$, Theorem 6.6; $\mathrm{GS}]$ ). For each $\alpha$ in $\Delta$, one can choose constants $N_{\alpha, \beta}$ and vectors $E_{\alpha} \in \mathfrak{g}_{\alpha}$ and $H_{\alpha}$ in $i \mathfrak{t}_{0}$ such that

(1) $B\left(E_{\alpha}, E_{\beta}\right)=\delta_{\alpha,-\beta},\left[E_{\alpha}, E_{-\alpha}\right]=H_{\alpha}$,

(2) $B\left(H_{\alpha}, H\right)=\alpha(H)$ for all $H$ in $\mathfrak{t}$,

(3) $\left[E_{\alpha}, E_{\beta}\right]=0$ if $\alpha \neq-\beta$ or $\alpha+\beta \notin \Delta$,

(4) $\left[E_{\alpha}, E_{\beta}\right]=N_{\alpha, \beta} E_{\alpha+\beta}$ if $\alpha, \beta, \alpha+\beta \in \Delta$. The $N_{\alpha, \beta}$ are nonzero real constants such that $N_{-\alpha,-\beta}=-N_{\alpha, \beta}$; also $N_{-\alpha,-\beta}=N_{-\beta, \alpha+\beta}=N_{\alpha+\beta,-\alpha}$,

(5) $\bar{E}_{\alpha}=\varepsilon_{\alpha} E_{-\alpha}$, where $\varepsilon_{\alpha}=-1$ (resp. 1) if $\alpha$ is compact (resp. noncompact), and

(6) $N_{\alpha, \beta}^{2}=\frac{q(1+p)}{2}(\alpha, \alpha)$, where $\beta+n \alpha(-p \leq n \leq q)$ is the root string of $\alpha$ through $\beta$.

In accordance with (2), for $\lambda$ in $\mathfrak{t}^{*}$, we define $H_{\lambda}$ to be the unique element such that $\left(H_{\lambda}, H\right)=\lambda(H)$ for all $H$ in $\mathfrak{t}$.

It will be convenient to define sections directly rather than develop the preliminary geometric structures. Let $\left(\chi_{\lambda}, \mathbb{C}_{\lambda}\right)$ be a character of $T$ with differential $\lambda$. Then a smooth section of the homogeneous holomorphic line bundle $\mathcal{L}_{\lambda}$ is defined as a smooth function $f: G \rightarrow \mathbb{C}_{\lambda}$ such that $f(g t)=\chi_{\lambda}^{-1}(t) f(g)$ for all $g$ in $G$ and 
$t$ in $T$. Likewise a smooth $(0, k)$-form with values in $\mathcal{L}_{\lambda}$ is defined as a smooth function

$$
\omega: G \rightarrow \mathbb{C}_{\lambda} \otimes \wedge^{k} \mathfrak{n}^{*} \quad \text { with } \quad \omega(g t)=\left(\chi_{\lambda} \otimes A d\right)\left(t^{-1}\right)(\omega(g))
$$

for all $g$ in $G$ and $t$ in $T$. In addition, assume that

$$
(\lambda+\delta, \alpha)<0
$$

for all $\alpha$ in $\Delta^{+}$, where $\delta=\frac{1}{2} \sum_{\alpha>0} \alpha$.

In Theorem 0.1 the functional components of a harmonic form may be chosen to be matrix coefficients. In this way, the square-integrability property follows immediately from the Schur orthogonality relations for the discrete series.

The choice of such matrix coefficients is determined entirely by $\mathfrak{n}$-cohomology of the $K$-finite vectors of $V$. As a $(\mathfrak{g}, K)$-module, the $K$-finite vectors are equivalent to the cohomologically induced module $A^{\mathfrak{b}}(\lambda)$.

The techniques of this work are purely algebraic and depend crucially on two properties of $A^{\mathfrak{b}}(\lambda)$ for $\lambda$ satisfying (1.2):

(1) $A^{\mathfrak{b}}(\lambda)$ has infinitesimal character parameter $\lambda+\delta$, and

(2) the lowest weight of the minimal $K$-type is $\lambda+2 \delta_{c}$.

If $\phi$ is a nonzero lowest weight vector of the minimal $K$-type of $V$, then $\phi^{\prime}=\langle\cdot, \phi\rangle$ is a highest weight vector of the minimal $K$-type of $V^{*}$ with weight $-\lambda-2 \delta_{c}$.

Let $\left\{E_{i}\right\}$ be any basis for $\mathfrak{g}$, and let $\left\{E^{i}\right\}$ be the corresponding dual basis of $\mathfrak{g}$ with respect to the Killing form. Then the Casimir operator $\Omega$ is defined in $U(\mathfrak{g})$, the universal enveloping algebra of $\mathfrak{g}$, by $\sum E_{i} E^{i}$, and this element lies in the center of $U(\mathfrak{g})$. Using the infinitesimal character property,

$$
\Omega \cdot v=((\lambda+\delta, \lambda+\delta)-(\delta, \delta)) v=((\lambda, \lambda)+2(\lambda, \delta)) v
$$

for $v$ in $V_{K-f i n}$.

\section{THE QUATERNIONIC POSITIVE ORDERING}

We consider only discrete series representations associated to certain real forms; these orderings were first used by Wolf $[\mathrm{Wo}$ ] in the classification of compact symmetric spaces with a quaternionic structure. See also $[\mathrm{Al}]$. For finer details for such representations, see [GW] and [Go].

Assume $\mathfrak{g}$ is simple, and let $\beta_{0}$ be the highest root of $\Delta^{+}$. There exists a real form $\mathfrak{g}_{0}$ of $\mathfrak{g}$ with

$$
\Delta_{c}=\left\{\beta \in \Delta \mid \beta \perp \beta_{0}\right\} \cup\left\{ \pm \beta_{0}\right\}
$$

as the set of compact roots, and

$$
\Delta_{n}=\left\{\beta \in \Delta \mid\left(\beta, \beta_{0}\right) \neq 0\right\}
$$

as the set of noncompact roots. We denote the associated subsets of positive roots by $\Delta_{c}^{+}, \Delta_{n}^{+}$, and define $\delta_{c}, \delta_{n}$ to be half the sum of the elements in these sets. The only positive compact root not in the span of the compact simple roots is $\beta_{0}$.

The Vogan diagram $([\mathrm{Kn}], \mathrm{Ch}$. VI $)$ is easily inferred from the extended Dynkin diagram; see Table 2.5 of [GW]. The Vogan diagram of such an ordering has no arrows, and one paints the nodes attached to $-\beta_{0}$. One sees that the multiplicity of a noncompact simple root in $\beta_{0}$ is 1 or 2 . Thus the multiplicity of a noncompact simple root in a positive noncompact root is 1 , except in the case of type $A$; in this case, the sum of the multiplicities of the two noncompact simple roots is 1 . 
Hence $\left(\beta_{0}, \alpha\right)$ is positive and independent of the positive noncompact root $\alpha$. Since $[\mathfrak{k}, \mathfrak{p}] \subset \mathfrak{p}$,

Lemma 2.1. The map $\alpha \mapsto \beta_{0}-\alpha$ is an involution of $\Delta_{n}^{+}$without fixed points.

It follows that $2 \delta_{n}=m \beta_{0}$, where $m=\frac{1}{2}\left|\Delta_{n}^{+}\right|$, and that $\left(2 \delta_{c}, 2 \delta_{n}\right)=m\left(\beta_{0}, \beta_{0}\right)$.

\section{The LaPLaCian}

Let $\omega^{-\alpha}$ be the basis for $\mathfrak{n}^{*}$ dual to $\left\{E_{-\alpha}\right\}$. To each ordered set $A=\left(\alpha_{1}, \ldots, \alpha_{k}\right)$ of $k$ distinct positive roots, we define $|A|=\sum \alpha_{i}$, and

$$
\omega^{-A}=\omega^{-\alpha_{1}} \wedge \cdots \wedge \omega^{-\alpha_{k}} .
$$

For $\alpha \in \Delta^{+}$, define

$$
i\left(\omega^{-\alpha}\right) \omega^{-A}= \begin{cases}0 & \text { if } \alpha \notin A, \\ \omega^{-A^{\prime}} & \text { if } \omega^{-A}=\omega^{-\alpha} \wedge \omega^{-A^{\prime}} .\end{cases}
$$

Then the explicit formula ([GS], Proposition 5.1) for the Laplacian on a monomial $(0, s)$-form is given by

$$
\begin{aligned}
\square\left(f \omega^{-A}\right)= & \frac{1}{2}(|A|, 2 \delta-|A|) f \omega^{-A} \\
& +\sum_{\alpha} \varepsilon_{\alpha}\left(H_{\alpha} f \omega^{-\alpha} \wedge i\left(\omega^{-\alpha}\right) \omega^{-A}-E_{\alpha} E_{-\alpha} f \omega^{-A}\right) \\
& +\sum_{\alpha, \beta}\left(\varepsilon_{\beta}-\varepsilon_{\alpha}\right) E_{\alpha} f A d\left(E_{-\alpha}\right) \omega^{-\beta} \wedge i\left(\omega^{-\beta}\right) \omega^{-A} \\
& +\sum_{\alpha, \beta}\left(\varepsilon_{\beta}+1\right) E_{-\alpha} f A d\left(E_{\alpha}\right) \omega^{-\beta} \wedge i\left(\omega^{-\beta}\right) \omega^{-A} .
\end{aligned}
$$

If $f$ represents instead an element of $V^{*},(3.1)$ also gives the associated Laplacian for the $\mathfrak{n}$-cohomology of $V^{*}$ in degree $s$.

Let $A$ be any ordered set of $s$ distinct positive roots containing all compact positive roots except possibly $\beta_{0}$. Then the Laplacian affects $\omega^{-A}$ only in the term not associated to the span of the compact simple roots. Thus, we consider only ordered sets $A$ where the first element is variable, and the remainder is a fixed listing of $\Delta_{c}^{+}-\left\{\beta_{0}\right\}$. For $\alpha$ in $\Delta_{n}^{+} \cup\left\{\beta_{0}\right\}$, denote by $\omega_{-\alpha}$ the corresponding exterior $s$-form with $\alpha$ as the first element of $A$.

It is now possible to state the main result:

Theorem 3.2. $\left(\square^{2}+\left((1-m) \beta_{0}+2 \lambda, \beta_{0}\right) \square\right) \phi^{\prime} \omega_{-\beta_{0}}=0$, and

$$
\omega_{\square}=\left(\square+\left((1-m) \beta_{0}+2 \lambda, \beta_{0}\right)\right) \phi^{\prime} \omega_{-\beta_{0}}
$$

defines a nonzero harmonic representative for $H^{s}\left(\mathfrak{n},\left(V^{*}\right)_{K-f i n}\right)_{-\lambda}$.

The proof occupies the next section. 
Corollary 3.3. The map

$$
\begin{gathered}
S: V \rightarrow \mathcal{H}_{2}^{0, s}\left(G / T, \mathcal{L}_{\lambda}\right), \\
(S v)(g)=\left(2 \lambda+\beta_{0}, \beta_{0}\right)\left\langle\pi(g)^{-1} v, \phi\right\rangle \omega_{-\beta_{0}}+2 \sum_{\alpha \in \Delta_{n}^{+}} N_{\beta_{0},-\alpha}\left\langle\pi(g)^{-1} v, E_{\alpha} \phi\right\rangle \omega_{-\beta_{0}+\alpha}
\end{gathered}
$$

defines a unitary equivalence (up to a scalar).

Proof. This follows immediately from Lemma 4.1 below.

\section{THE PROOF}

A general approach to finding explicit harmonic classes for $\mathfrak{n}$-cohomology is given as follows. Suppose that there exists an element $\omega_{s}$ in $V^{*} \otimes \wedge^{s} \mathfrak{n}^{*}$ such that its cyclic span under $\square$ is finite-dimensional, and let $p(x)$ be the monic polynomial of smallest degree such that $p(\square) \omega_{s}=0$. If one can write $p(x)=x q(x)$, then $\omega_{\square}=q(\square) \omega_{s}$ is a nonzero harmonic form. Consideration [Do of the Penrose transform and the Borel-Weil-Bott theorem suggest that a suitable candidate for $\omega_{s}$ is given by $\phi^{\prime} \omega^{-A}$, where $A$ is the set of positive compact roots.

It is instructive to first consider the holomorphic discrete series. In this case, the positive ordering is derived from a Vogan diagram with no arrows and a single painted node; the multiplicity of the corresponding noncompact simple root in the highest root is 1 . This ordering corresponds to a good ordering; that is, every positive noncompact root is greater than every compact root. In addition, all compact roots lie in the span of the compact simple roots. Earlier considerations imply that lines 2 and 3 of (3.1) vanish, and one sees by modifying the proof of Lemma 4.1 below that $\omega_{s}$ is itself harmonic.

Now assume that the assumptions of section 2 hold.

Lemma 4.1. One has

$$
\square\left(\phi^{\prime} \omega_{-\beta_{0}}\right)=m\left(\beta_{0}, \beta_{0}\right) \phi^{\prime} \omega_{-\beta_{0}}+2 \sum_{\alpha \in \Delta_{n}^{+}} N_{\beta_{0},-\alpha} E_{\alpha} \phi^{\prime} \omega_{-\beta_{0}+\alpha} .
$$

Proof. Note that only the first three lines of (3.1) contribute. The first term of (3.1) equals $\frac{1}{2}\left(2 \delta_{c}, 2 \delta_{n}\right)=\frac{m}{2}\left(\beta_{0}, \beta_{0}\right)$. Next

$$
\sum_{\alpha \in \Delta_{c}^{+}} H_{\alpha} \phi^{\prime}=-\left(\lambda+2 \delta_{c}, 2 \delta_{c}\right) \phi^{\prime} .
$$

In $U(\mathfrak{g})$, one has

$$
-\sum_{\alpha \in \Delta^{+}} \varepsilon_{\alpha} E_{\alpha} E_{-\alpha}=2 \sum_{\alpha \in \Delta_{c}^{+}} E_{\alpha} E_{-\alpha}-\sum_{\alpha \in \Delta^{+}} E_{\alpha} E_{-\alpha} .
$$

For $\alpha$ in $\Delta_{c}^{+}, E_{\alpha} E_{-\alpha} \phi^{\prime}=E_{-\alpha} E_{\alpha} \phi^{\prime}+H_{\alpha} \phi^{\prime}=-\left(\lambda+2 \delta_{c}, \alpha\right) \phi^{\prime}$. In addition, the second sum equals $\frac{1}{2} \Omega+H_{\delta}-\frac{1}{2} \sum H_{i}^{2}$, where $\Omega$ is the Casimir element and $\left\{H_{i}\right\}$ is an orthonormal basis for $\mathfrak{t}$. Applied to $\phi^{\prime}$, we obtain

$$
\begin{aligned}
\left(\frac{1}{2}((\lambda, \lambda)+2(\lambda, \delta))\right. & \left.-\left(\lambda+2 \delta_{c}, \delta\right)-\frac{1}{2}\left(\lambda+2 \delta_{c}, \lambda+2 \delta_{c}\right)\right) \phi^{\prime} \\
& =\left(-\left(2 \delta_{c}, \delta\right)-\left(\lambda, 2 \delta_{c}\right)-\left(\delta_{c}, 2 \delta_{c}\right)\right) \phi^{\prime} .
\end{aligned}
$$

Combining these terms as indicated, the lemma follows. 
Definition 4.2. For $\alpha$ in $\Delta_{n}^{+}$, define $\Delta(\alpha)=\left\{\gamma \in \Delta_{c}^{+} \mid \alpha+\gamma \in \Delta_{n}^{+}\right\}$.

Lemma 4.3. For $\alpha$ in $\Delta_{n}^{+}$, one has

$$
\begin{aligned}
\square\left(E_{\alpha} \phi^{\prime} \omega_{-\beta_{0}+\alpha}\right) & =2 \sum_{\gamma \in \Delta(\alpha)} N_{\gamma, \alpha} E_{-\gamma} E_{\gamma+\alpha} \phi^{\prime} \omega_{-\beta_{0}+\alpha} \\
& +\left((m-2)(\beta-\alpha, \beta)+\left(2 \delta_{c}-\alpha, \alpha\right)-2\left(\lambda, \beta_{0}\right)\right) E_{\alpha} \phi^{\prime} \omega_{-\beta_{0}+\alpha} \\
& -2 \sum_{\gamma \in \Delta(\alpha)} N_{\beta_{0}-\alpha, \gamma} E_{-\gamma} E_{\alpha} \phi^{\prime} \omega_{-\beta_{0}+\alpha-\gamma} \\
& -2 \sum_{\gamma \in \Delta(\alpha)} N_{\beta_{0}-\alpha,-\gamma} N_{\gamma, \alpha} E_{\gamma+\alpha} \phi^{\prime} \omega_{-\beta_{0}+\alpha+\gamma} \\
& -2 N_{\beta_{0},-\alpha} E_{-\alpha} E_{\alpha} \phi^{\prime} \omega_{-\beta_{0}} .
\end{aligned}
$$

Proof. The argument is identical to Lemma 4.1. The first line arises from application of $-2 \sum_{\alpha \in \Delta_{c}^{+}} E_{-\alpha} E_{\alpha}$ and use of the highest weight property of $\phi^{\prime}$. To determine the second line, note the following:

$$
\begin{gathered}
\frac{1}{2}(|A|, 2 \delta-|A|)=\frac{1}{2}\left(2 \delta_{c}-\alpha, 2 \delta_{n}+\alpha\right), \\
\left(-H_{2 \delta_{c}-\beta_{0}}+H_{\beta_{0}-\alpha}\right) E_{\alpha} \phi^{\prime}=\left(\lambda+2 \delta_{c}-\alpha, 2 \delta_{c}-2 \beta_{0}+\alpha\right) E_{\alpha} \phi^{\prime}, \\
2 H_{2 \delta_{c}} E_{\alpha} \phi^{\prime}=-2\left(\lambda+2 \delta_{c}-\alpha, 2 \delta_{c}\right) E_{\alpha} \phi^{\prime},
\end{gathered}
$$

and

$$
\begin{aligned}
\left(\frac{1}{2} \Omega+H_{\delta}-\frac{1}{2} \sum H_{i}^{2}\right) E_{\alpha} \phi^{\prime}= & \left(\frac{1}{2}(\lambda, \lambda)+(\lambda, \delta)-\left(\lambda+2 \delta_{c}-\alpha, \delta\right)-\frac{1}{2}(\lambda, \lambda)\right. \\
& \left.-\left(\lambda, 2 \delta_{c}-\alpha\right)-\frac{1}{2}\left(2 \delta_{c}-\alpha, 2 \delta_{c}-\alpha\right)\right) E_{\alpha} \phi^{\prime} \\
= & -\left(\lambda+\delta+\delta_{c}-\frac{1}{2} \alpha, 2 \delta_{c}-\alpha\right) E_{\alpha} \phi^{\prime}
\end{aligned}
$$

Combining these terms as indicated gives the second term of the equation.

Lemmas 4.1 and 4.3 yield

$$
\begin{aligned}
\square^{2}\left(\phi^{\prime} \omega_{-\beta_{0}}\right) & =m^{2}\left(\beta_{0}, \beta_{0}\right)^{2} \phi^{\prime} \omega_{-\beta_{0}}+2 m\left(\beta_{0}, \beta_{0}\right) \sum_{\alpha \in \Delta_{n}^{+}} N_{\beta_{0},-\alpha} E_{\alpha} \phi^{\prime} \omega_{-\beta_{0}+\alpha} \\
& +4 \sum_{\alpha^{\prime} \in \Delta_{n}^{+}} \sum_{\gamma \in \Delta\left(\alpha^{\prime}\right)} N_{\beta_{0},-\alpha^{\prime}} N_{\gamma, \alpha^{\prime}} E_{-\gamma} E_{\gamma+\alpha^{\prime}} \phi^{\prime} \omega_{-\beta_{0}+\alpha^{\prime}} \\
& -4 \sum_{\alpha \in \Delta_{n}^{+}} \sum_{\gamma \in \Delta(\alpha)} N_{\beta_{0},-\alpha} N_{\beta_{0}-\alpha, \gamma} E_{-\gamma} E_{\alpha} \phi^{\prime} \omega_{-\beta_{0}+\alpha-\gamma} \\
& -4 \sum_{\alpha \in \Delta_{n}^{+}} \sum_{\gamma \in \Delta(\alpha)} N_{\beta_{0},-\alpha} N_{\beta_{0}-\alpha,-\gamma} N_{\gamma, \alpha} E_{\gamma+\alpha} \phi^{\prime} \omega_{-\beta_{0}+\alpha+\gamma} \\
& +2 \sum_{\alpha \in \Delta_{n}^{+}} N_{\beta_{0},-\alpha} C_{\lambda, \alpha} E_{\alpha} \phi^{\prime} \omega_{-\beta_{0}+\alpha} \\
& -4 \sum_{\alpha \in \Delta_{n}^{+}} N_{\beta_{0},-\alpha}^{2} E_{-\alpha} E_{\alpha} \phi^{\prime} \omega_{-\beta_{0}},
\end{aligned}
$$


where

$$
C_{\lambda, \alpha}=(2 m-2)\left(\beta_{0}-\alpha, \beta_{0}\right)+\left(2 \delta_{c}-\alpha, \alpha\right)-2\left(\lambda, \beta_{0}\right) .
$$

For the remainder of this section, a battery of lemmas will be used to simplify this sum.

Lemma 4.5. For $\alpha$ in $\Delta_{n}^{+}$and $\gamma$ in $\Delta(\alpha)$,

$$
N_{\beta_{0}, \gamma-\alpha} N_{\gamma, \alpha-\gamma}=N_{\beta_{0},-\alpha} N_{\beta_{0}-\alpha, \gamma} .
$$

Proof. Note that

$$
\begin{aligned}
N_{\beta_{0},-\alpha} N_{\beta_{0}-\alpha, \gamma} E_{\beta_{0}-\alpha+\gamma} & =\left[\left[E_{\beta_{0}}, E_{-\alpha}\right], E_{\gamma}\right] \\
& =-\left[\left[E_{-\alpha}, E_{\gamma}\right], E_{\beta_{0}}\right] \\
& =-N_{-\alpha, \gamma} N_{-\alpha+\gamma, \beta_{0}} E_{\beta_{0}-\alpha+\gamma} .
\end{aligned}
$$

Since $\beta_{0}+\gamma$ is not a root, the second equality follows from the Jacobi identity. Using Proposition 1.1(4), $N_{-\alpha, \gamma}=N_{\gamma, \alpha-\gamma}$ and $N_{-\alpha+\gamma, \beta_{0}}=-N_{\beta_{0}, \gamma-\alpha}$, and the lemma follows.

Thus the second and third lines of (4.4) sum to zero after aligning indices by $\alpha^{\prime}=\alpha-\gamma$.

The next two lemmas simplify the fourth line of (4.4).

Lemma 4.6. For $\alpha$ in $\Delta_{n}^{+}$,

$$
\sum_{\gamma \in \Delta(\alpha)} N_{\beta_{0}, \gamma-\alpha} N_{\beta_{0}-\alpha+\gamma,-\gamma} N_{\gamma, \alpha-\gamma}=N_{\beta_{0},-\alpha} \sum_{\gamma \in \Delta(\alpha)} N_{\beta_{0}-\alpha, \gamma}^{2} .
$$

Proof. Since $N_{\gamma, \alpha-\gamma}=N_{-\alpha, \gamma}$, the left-hand side equals

$$
\sum_{\gamma \in \Delta(\alpha)} B\left(\left[\left[E_{\beta_{0}},\left[E_{-\alpha}, E_{\gamma}\right]\right], E_{-\gamma}\right], E_{-\beta_{0}+\alpha}\right) .
$$

Since $\beta_{0}+\gamma$ is not a root, the Jacobi identity implies that

$$
\begin{aligned}
{\left[\left[E_{\beta_{0}},\left[E_{-\alpha}, E_{\gamma}\right]\right], E_{-\gamma}\right] } & =-\left[\left[E_{\gamma},\left[E_{\beta_{0}}, E_{-\alpha}\right]\right], E_{-\gamma}\right] \\
& =-N_{\beta_{0},-\alpha} N_{\gamma, \beta_{0}-\alpha} N_{\beta_{0}-\alpha+\gamma,-\gamma} E_{\beta_{0}-\alpha} .
\end{aligned}
$$

Since $N_{\beta_{0}-\alpha+\gamma,-\gamma}=N_{-\gamma, \alpha-\beta_{0}}=N_{\beta_{0}-\alpha, \gamma}$ and $N_{\gamma, \beta_{0}-\alpha}=-N_{\beta_{0}-\alpha, \gamma}$, the lemma follows.

Lemma 4.7. Fix $\alpha$ in $\Delta_{n}^{+}$. Then

$$
2 \sum_{\gamma \in \Delta(\alpha)} N_{\beta_{0}-\alpha, \gamma}^{2}=(m-1)\left(\beta_{0}, \beta_{0}\right)+(2-m)\left(\alpha, \beta_{0}\right)+\left(2 \delta_{c}-\alpha, \alpha\right) .
$$

Proof. Noting Proposition 1.1(6), we replace $(\cdot, \cdot)$ by a scalar multiple such that $\left(\beta_{0}, \beta_{0}\right)=2$.

First consider the simply-laced case. Then $(\beta, \beta)=2$ for all $\operatorname{roots} \beta$, and $\left|\left(\beta_{1}, \beta_{2}\right)\right|$ $=0$ or 1 for $\beta_{1} \neq \pm \beta_{2}$. In addition, orthogonality implies strong orthogonality; that is, if $\left(\beta_{1}, \beta_{2}\right)=0$, then $\pm \beta_{1} \pm \beta_{2}$ are not roots. By Proposition 1.1(6), for each $\gamma$ in $\Delta(\alpha), N_{\beta_{0}-\alpha, \gamma}^{2}=1$.

We prove the statement by induction on $\operatorname{ht}(\alpha)$, the height of $\alpha$. If $\operatorname{ht}(\alpha)=0$, then $\alpha$ is simple. In this case, the left-hand side is zero. The right-hand side equals

$$
(2 m-2)+(2-m)\left(\alpha, \beta_{0}\right)+\left(2 \delta-m \beta_{0}, \alpha\right)-(\alpha, \alpha) .
$$


Since $\alpha$ is simple for $\Delta^{+}(\mathfrak{g}), \frac{2(\delta, \alpha)}{(\alpha, \alpha)}=1$. Because $\left(\alpha, \beta_{0}\right)=1$ by the choice of positive ordering, the statement follows.

Suppose that equality holds for all $\alpha$ with height $n$ and that $\alpha+\gamma^{\prime}$ is a root, where $\alpha$ is a postive noncompact root of height $n$ and $\gamma^{\prime}$ is a compact simple root. Since $\frac{2\left(\delta_{c}, \gamma^{\prime}\right)}{\left(\gamma^{\prime}, \gamma^{\prime}\right)}=1$, the induction step follows if the equation

$$
\left|\Delta\left(\alpha+\gamma^{\prime}\right)\right|-|\Delta(\alpha)|=-\left(\gamma^{\prime}, \alpha\right)
$$

holds. If $\alpha^{\prime}+\gamma=\alpha$, then $-1=\left(\gamma^{\prime}, \alpha\right)=\left(\gamma^{\prime}, \alpha^{\prime}+\gamma\right)$, which implies that exactly one of $\alpha^{\prime}+\gamma^{\prime}$ or $\gamma+\gamma^{\prime}$ is a (noncompact (resp., compact)) root. Thus exactly one of $\left(\alpha^{\prime}+\gamma^{\prime}\right)+(\gamma)$ or $\left(\alpha^{\prime}\right)+\left(\gamma+\gamma^{\prime}\right)$ is a decomposition of $\alpha+\gamma^{\prime}$ contributing to $\Delta\left(\alpha+\gamma^{\prime}\right)$. Since $(\alpha)+\left(\gamma^{\prime}\right)$ is a decomposition of $\alpha+\gamma^{\prime}$ not arising in this manner, $\left|\Delta\left(\alpha+\gamma^{\prime}\right)\right| \geq|\Delta(\alpha)|+1$. A similar argument proves the reverse inequality, and the induction statement follows.

For the cases of types B and $\mathrm{C}$, the induction proof may be adapted. Short roots have $(\beta, \beta)=1$. Again $\left|\left(\beta_{1}, \beta_{2}\right)\right|=0$ or 1 for $\beta_{1} \neq \pm \beta_{2}$, and $N_{\beta_{0}-\alpha, \gamma}^{2}=1$. When $\alpha$ is a short simple root (type $\mathrm{C}$ ), the initial induction step still holds. To adapt the general induction step, assume $\left(\alpha, \gamma^{\prime}\right)=0$ and $\alpha+\gamma^{\prime}$ is a root. Since there are only two root lengths, $\alpha$ and $\gamma^{\prime}$ are short roots. Such a sum does not occur in type $\mathrm{C}$, and the lemma follows for type $\mathrm{C}$.

We assume the usual notation and standard ordering for type $\mathrm{B}_{n}, n \neq 2$. Then $\beta_{0}=e_{1}+e_{2}$ and

$$
\Delta_{n}^{+}=\left\{e_{1}, e_{2}, e_{1} \pm e_{i}, e_{2} \pm e_{i} \mid \text { all } i \neq 1,2\right\} .
$$

Here $\gamma^{\prime}=e_{n}$. Now $\left|\Delta\left(e_{1}\right)\right|=n-1$ since $\left(e_{1}-e_{j}\right)+\left(e_{j}\right)$ are the only desired decompositions of $e_{1}$. Similarly $\left(e_{1}-e_{j}\right)+\left(e_{j}+e_{n}\right)$ and $\left(e_{1}\right)+\left(e_{n}\right)$ yield all decompositions for $e_{1}+e_{n}$, and $\left|\Delta\left(e_{1}\right)\right|=\left|\Delta\left(e_{1}+e_{n}\right)\right|$. If $\alpha=e_{2}$, then $\left(e_{2}-e_{j}\right)+\left(e_{j}\right)$ gives all decompositions for $e_{2}$, and the decompositions for $e_{2}+e_{n}$ are given by $\left(e_{2}-e_{j}\right)+\left(e_{j}+e_{n}\right)$ and $\left(e_{2}\right)+\left(e_{n}\right)$. Thus $\left|\Delta\left(e_{2}\right)\right|=\left|\Delta\left(e_{2}+e_{n}\right)\right|$. It follows that the induction step holds in the case of type B.

We omit the proof for types $\mathrm{F}(m=7)$ and $\mathrm{G}(m=2)$. Using Proposition 1.1(6), these cases are easily verified directly from root tables (cf. [Kn], Appendix C).

Remark. For the classical cases, direct verification of Lemma 4.7 is rather straightforward.

The next two lemmas simplify the final line of (4.4).

Lemma 4.8. For all $\alpha$ in $\Delta_{n}^{+}, N_{\beta_{0},-\alpha}^{2}=\frac{1}{2}\left(\beta_{0}, \beta_{0}\right)$.

Proof. This follows immediately from Proposition 1.1(6). Since $-\alpha+\beta_{0}$ is a root but $-\alpha+2 \beta_{0}=\beta_{0}+\left(\beta_{0}-\alpha\right)$ is not, $q=1$. Since $-\alpha-\beta_{0}=-\left(\beta_{0}+\alpha\right)$ is not a root, $p=0$.

Lemma 4.9. $\sum_{\alpha \in \Delta_{n}^{+}} E_{-\alpha} E_{\alpha} \phi^{\prime}=\left(\lambda+\delta_{c}, 2 \delta_{n}\right)=m\left(\lambda, \beta_{0}\right)+\frac{m}{2}\left(\beta_{0}, \beta_{0}\right)$.

Proof. Since $E_{\gamma} \phi^{\prime}=0$ for $\gamma$ in $\Delta_{c}^{+}$, the sum may be taken over all positive roots. In $U(\mathfrak{g})$, one has

$$
\sum_{\alpha \in \Delta} E_{-\alpha} E_{\alpha}=\frac{1}{2} \Omega-H_{\delta}-\frac{1}{2} \sum H_{i}^{2}
$$

where $H_{i}$ runs over an orthonormal basis of $\mathfrak{h}_{0}$. A computation as in Lemma 4.1 proves the lemma. 
Proof of Theorem 3.2. Using the above simplifications to recalculate (4.4), one obtains

$$
\begin{aligned}
& \square^{2}\left(\phi^{\prime} \omega_{-\beta_{0}}\right) \\
= & \left((m-1)\left(\beta_{0}, \beta_{0}\right)-2\left(\lambda, \beta_{0}\right)\right)\left(m\left(\beta_{0}, \beta_{0}\right) \phi^{\prime} \omega_{-\beta_{0}}+2 \sum_{\alpha \in \Delta_{n}^{+}} N_{\beta_{0},-\alpha} E_{\alpha} \phi^{\prime} \omega_{-\beta_{0}+\alpha}\right) .
\end{aligned}
$$

Hence the theorem follows from Lemma 4.1.

\section{ACKNOWLEDGMENTS}

The author completed this work as a participant in the Integral Geometry Program (Fall 2001) at the Mathematical Sciences Reaserch Institute in Berkeley. The author thanks Roger Zierau for helpful conversations, and further useful comments were made by Leticia Barchini, Paul Friedman, Anthony Knapp, and Joe Wolf.

\section{REFERENCES}

[Al] D. V. Alekseevskii, Compact quaternion spaces, Functional Anal. and Its Appl. 2 (1968), 106-114. MR 37:6869 (Russian)

[Ba] L. Barchini, Strongly harmonic forms for representations in the discrete series, J. Funct. Anal. 161 (1999), 111-131. MR 2000a:22019

[Do] R. W. Donley, Jr., Orthogonality relations and harmonic forms for semisimple Lie groups, J. Funct. Anal. 170 (2000), 141-160. MR 2001j:22016

[Go] D. Gordon, Quaternionic discrete series, Represent. Theory 3 (1999), 32-57. MR 2001g:22025

[GS] P. Griffiths, and W. Schmid, Locally homogeneous complex manifolds, Acta Math. 123 (1969), 253-302. MR 41:4587

[GW] B. H. Gross, and N. R. Wallach, On quaternionic discrete series representations, and their continuations, J. Reine Angew. Math. 481 (1996), 73-123. MR 98f:22022

[HC] Harish-Chandra, Discrete series for semisimple Lie groups. II. Explicit determination of the characters, Acta Math. 116 (1966), 1-111. MR 36:2745

[He] S. Helgason, Differential Geometry and Symmetric Spaces, Academic Press, New York, 1962. MR 26:2986

[Kn] A. W. Knapp, Lie Groups Beyond an Introduction, Birkhäuser, Boston, 1996. MR 98b:22002

[Sc] W. Schmid, L2 -cohomology and the discrete series, Ann. of Math. 103 (1976), 375-394. MR 53:716

[Wo] J. A. Wolf, Complex homogeneous contact manifolds and quaternionic symmetric spaces, J. Math. and Mech. 14 (1965), 1033-1047. MR 32:3020

Department of Mathematics, University of North Texas, Denton, Texas 76203

E-mail address: rdonley@unt.edu 